\title{
Maximum-Utility Scheduling for Multimedia Transmission in Drive-Thru Internet
}

\author{
Min Xing, Student Member, IEEE, Jianping He, and Lin Cai, Senior Member, IEEE
}

\begin{abstract}
How to support multimedia services for people on the road is a pressing issue. Relying on the vehicle to infrastructure (V2I) communications, the limited wireless resources and vehicle sojourn time make it quite challenging to schedule the transmissions of multiple vehicles to ensure high efficiency and quality. In this paper, the scheduling of multimedia transmissions over Drive-thru Internet is investigated. A utility model is devised to map the throughput to user's satisfaction level. The objective of the scheduling problem is to maximize the total utility. Then the optimization problem is formulated as a finite-state decision problem with the assumption that future arrival information is known, and it is solved by a searching algorithm as the benchmark. To obtain a real-time solution, a practical heuristic algorithm based on the concept of utility potential is devised. We further implemented the solution and conducted extensive simulations using NS-3, and the simulation results show that the proposed heuristic algorithm can outperform the state-of-the-art one, so it can effectively make scheduling decisions to achieve both higher utility and efficiency, and better fairness.
\end{abstract}

Index Terms-Multimedia transmission, Utility, Drive-thru Internet.

\section{INTRODUCTION}

$\mathbf{T}$ HERE is an increasing demand of Internet service nowadays, even for people in the moving vehicles. Due to the high mobility for them, it is quite challenging to provide high-speed, low-cost and reliable Internet services. Vehicle to infrastructure communications (V2I) is a promising solution to cope with the mobility challenge. Road Side Units (RSU) are deployed along the road to act as Internet access points (AP). When vehicles pass through the coverage area of a RSU, they can access the Internet by connecting to the RSU. The above system, termed Drive-thru Internet, has attracted many research interests recently [1]-[3].

Multimedia applications in Drive-thru Internet have emerged recently [4]-[7]. For instance, video and voice are desirable media to deliver advertisements, news and etc. to improve the experience of passengers. Meanwhile, multimedia transmission can also take an important role to enhance road safety [8]. For example, video clips of dangerous road conditions taken by the vehicles ahead can assist overtaking on rural roads [9]. However, the transmission of videos and other multimedia traffic over Drive-thru Internet can be quite challenging. Due to mobility, the sojourn time of the vehicle in the RSU's coverage range is limited which restricts the total amount of data that can be transmitted. When multiple vehicles

M. Xing, J. He and L. Cai are with the Department of Electrical and Computer Engineering, University of Victoria, Victoria, BC, Canada, V8W 3P6. (e-mail: mxing@ece.uvic.ca, jphe@uvic.ca, cai@ece.uvic.ca). The corresponding author is Jianping He. are within the coverage of a single RSU, the competing of limited bandwidth will further reduce the throughput of each vehicle. Therefore, the scheduling problem of multimedia transmission in Drive-thru Internet is an open issue.

In this paper, the scheduling problem of multimedia traffic including video, voice and data, among multiple vehicles accessing a single RSU is investigated. Videos encoded with various techniques are taken as sample applications in our work, and other kinds of multimedia traffic can be easily incorporated to our approach as well. Motivated by [10], a time division multiplex access (TDMA)-based scheduling algorithm is devised to maximize the total utility of the transmitted videos. The utility model is devised as a function to map the total flow throughput to user's satisfaction level, such as the decoded quality of video. Channel time will be divided into time slots. For each time slot, only one vehicle will be allocated to transmit. The scheduling problem is then formulated as an optimization problem to maximize the total achieved utility. The optimal solution is difficult to obtain since the solution depends on the future arrivals, and the optimal decision cannot be simply decoupled into per-slot optimization problems. To solve this challenging issue, we propose a heuristic algorithm based on the concept of utility potential.

The main contributions of this paper are summarized as follows. First, considering the new problem of scheduling heterogeneous multimedia flows in Drive-thru Internet, we define utility models to map the throughput to user's satisfaction level, and formulate the optimization problem to maximize the total utility. Second, by converting the optimization problem into a finite-state decision problem with the assumption that the future arrivals are known, the optimal results can be obtained by a searching algorithm. As the assumption is not realistic and the searching algorithm is too complicated to use, we propose a heuristic algorithm based on the utility potential to make scheduling decisions in real time. Finally, we implement the proposed scheduling algorithm and conduct extensive simulations using NS-3 [11] to evaluate its performance. The results show that the proposed heuristic algorithm can substantially outperform the state-of-the-art one [1] in terms of total utility, resource utilization, and fairness.

The rest of the paper is organized as follows. In Section II, the related work is summarized. Section III describes the system model and the problem formulation. We present the optimal upper bound and scheduling algorithm in Section IV. The performance evaluation by simulation is presented in Section $\mathrm{V}$, followed by the concluding remarks and further research issues in Section VI. 


\section{RELATED WORK}

Vehicle to infrastructure communications (V2I) has attracted research interests in recent years, and there are extensive work studying the Drive-thru communication scenarios [1]-[3], [12]-[16]. Tan et al. proposed an analytical model in [12] to characterize the downlink average throughput and distribution achieved for each vehicle during the sojourn time by a Markov reward model, with the assumption that the wireless resource is evenly shared by all the passing vehicles. In [13], the authors tried to model the uplink performance of the last-hop Drivethru communication with the consideration of vehicle density and speed. They showed that with an optimal admission control scheme, the throughput can be maximized for each Drive-thru vehicle. For the non-real-time traffic transmission of V2I communication, Alcaraz et al. proposed a contentionfree, poll-based link-layer scheduling algorithm [14], aiming to reduce the residual queue backlog for each user by assigning the user with the lower packet error rate a higher priority. Cheung et al. studied the random access problem in the Drive-thru scenario [2], [15]. They formulated the optimal transmission problem as a finite-horizon sequential decision problem and solved it by dynamic programming. Due to the high computational complexity, only an offline solution was obtained. Zhang et al. proposed an application-layer service scheduling algorithm with the consideration of both service deadline and data size [1]. The variation of communication distance was ignored in their work and a constant transmission rate was assumed.

Recently, utility-based TDMA scheduling has been applied in many works [10], [17]-[23]. Considering the mobility pattern of unmanned aircraft system, [17] formulated the data collection problem as a potential game between the unmanned aircraft and the ground nodes to maximize the energy efficiency of the ground nodes. How to improve both spectrum and energy efficiency was considered in [18]. Utility-based flow control optimization problems were formulated for wireless sensor networks with lifetime constraint [20], [21]. In [10], the problem of utility maximization of concurrent transmission scheduling in UWB networks was studied. The definition of utility function depends on the traffic type. By modeling the network into a graph, a heuristic scheduling algorithm based on the "exclusive region" was proposed. Similarly, Hwang et al. considered the video multicast problem in wireless mesh networks which was also modeled into graphs [19]. By jointly considering routing and scheduling the transmissions over the graph, the system utility was maximized. In [22], the optimal utility scheduling of adaptive video streaming over a small cell network was formulated into an optimization problem and solved by liner programming. Scheduling multimedia applications over heterogeneous wireless networks was investigated in [23], and a novel distributed approach was proposed to maximize the utility which jointly considered QoS, reliability and availability. However, the mobility of nodes was not considered in the above work, and we cannot apply or extend the above solutions easily to support multimedia services in Drive-thru network, which motivated this work.

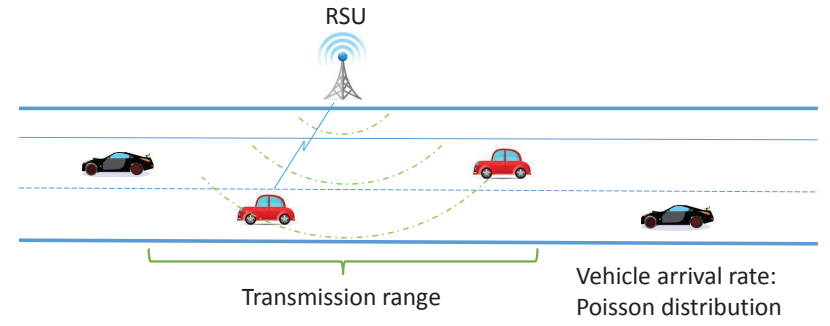

Fig. 1. System Model.

\section{System Model ANd Problem Formulation}

We consider how to schedule multiple vehicles to transmit the multimedia data in a highway Drive-thru scenario, which is shown in Fig. 1. In our scenario, both uplink and downlink are considered, such that each vehicle may request or upload multimedia data through V2I communications to RSU. The $\mathrm{RSU}$ is deployed on the road side with the transmission range of $R$. Due to the high cost, it is impossible to deploy enough RSUs to cover the entire road, and each RSU only covers a certain range. As a result, each vehicle moving on the highway can transmit multimedia data only when it passes a RSU. As mentioned before, videos encoding with various techniques are considered as examples in this work, and other multimedia traffic, such as voice, image, data, and etc., can also be incorporated into our approach easily. For each vehicle, we consider only one video flow will be carried, and it will be encoded by one encoding technique. The encoded video will be chopped into small packets for transmission. A vehicle is allowed to transmit only during its allocated time slots. When the multimedia data is successfully transmitted, it does not request wireless network access any more.

\section{A. Vehicle Mobility Model}

According to the vehicle traffic model [13], [24], the arrival process of vehicles can be modeled as a Poisson distribution process. The arrival rate of the vehicles for each lane is assumed to be $\lambda$ (number of vehicles per time unit). With multiple lanes in both directions, the aggregated arrival still follows a Poisson distribution. To simplify the notation in the following, we assume that all vehicles are in a single lane and the same approach can be applied to multiple lanes cases.

With the consideration of a speed limit in highway, it is reasonable to assume that all vehicles are moving with the average speed $v$ (distance traveled per time unit). Let $k$ represent the vehicle density (number of vehicles per kilometer), and $k=\lambda / v$. Then the average number of vehicles passing an observation point per time unit is $q=k v$. According to [24], the relationship between vehicle density and speed can be modeled as:

$$
v=v_{f}\left(1-k / k_{\max }\right),
$$

where $v_{f}$ is the free-flow speed and $k_{\max }$ is the vehicle jam density. With the vehicle's speed $v$, the total duration of vehicle with the coverage range of the RSU is easy to obtain:

$$
T_{s}=2 R / v
$$




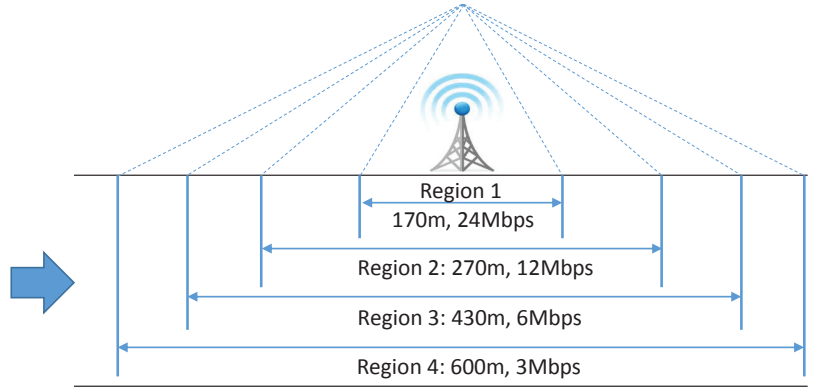

Fig. 2. Wireless Model.

where $R$ is the transmission range of the RSU. The number of vehicles in the coverage range of the RSU can also be calculated:

$$
N_{v}=\left\lfloor T_{s} k v\right\rfloor .
$$

\section{B. Wireless Model}

As we focus on the resource allocation in a general wireless system that can support transmission scheduling, we consider a general wireless communication model, which suffers from fading, shadowing and path loss. It is possible that the RSU uses cellular or WiFi technologies. If using the cellular technology, the TDMA mode can support the proposed scheduling directly; if using WiFi with contention-based MAC, then we may rely on the association process to deploy the proposed scheduling algorithm.

Broadband wireless systems typically support scalable modulation and coding techniques to adjust the data rate according to the received signal to noise ratio (SNR). Further, the wireless channel quality between the RSU and the moving vehicle highly depends on the path loss which is a function of the communication distance $d$. Thus, we can simplify the wireless model by mapping the transmission distance to the data rate. Although shadowing and fading may also affect the instantaneous signal strength and should be considered for the data communication, but this part is not considered by the scheduler to reduce the control overhead and simplify the scheduling decision. This strategy is also adopted by references [2], [12]. Therefore, the coverage area of the RSU is divided into several regions, which is shown in Fig. 2. Each region has a constant transmission data rate. The closer to the RSU, the higher the data rate it supports.

\section{Utility Model}

Different from the previous work, we consider different utility functions for different types of video encoding techniques. As a widely accepted video quality metric, Peak Signal-toNoise Ratio (PSNR) is used to represent the value of utility function [25], [26]. The utility function $F_{u}$ maps the amount of transmitted video data to the decoded quality, which can be represented by:

$$
U=F_{u}^{j}(D), j=\left\{1,2, \ldots, N_{e}\right\},
$$

where $D$ is the amount of transmitted video data, $N_{e}$ is the total number of encoding techniques. For each video, with a

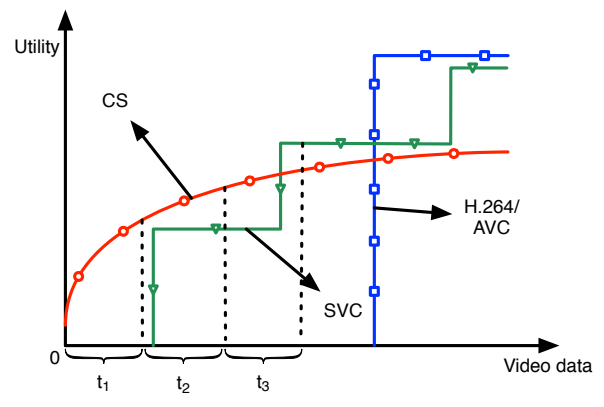

Fig. 3. Utility Model.

different encoding technique, the required amount of data to decode is different. For example, for the H.264/AVC codec, only if all the video packets are received successfully, the video can be decoded. While for compressed sensing (CS) based encoding technique, the video can always be decoded with different quality depending on the amount of received data. Therefore, the utility function can be either discrete or continuous functions. For instance, the utility function $F_{u}$ for H.264/AVC codec can be defined as:

$$
F_{u}= \begin{cases}u, & \text { if } D \geq S_{v}, \\ 0, & \text { otherwise }\end{cases}
$$

where $S_{v}$ is the total size of the encoded video clips. The above discrete utility function is shown as the blue curve with square marker in Fig. 3. Similarly, for Scalable Video Coding (SVC), as the video is encoded into layers, with more video data transmitted, more enhancement layers can be decoded to improve the video quality. Thus, the utility function for SVC is a stair-case discrete function, as the green curve with triangle marker shown in Fig. 3. While for the CS based encoding technique, since the video can always be decoded with any amount of received data, the utility function can be written as a quadratic function [26], [27]:

$$
F_{u}=a D^{b}+c,
$$

where $a, b$ and $c$ are the parameters determined by the encoder, shown as the red curve with circle marker in Figure 3.

\section{Problem Formulation}

As there is a speed limit for highway, it is reasonable to assume that all the vehicles move with the average speed $v$. Similar to the typical MAC approach, the total sojourn time can be divided into time slots with the duration of $\Delta t$. When the time slot $\Delta t$ is small enough, there will be at most one vehicle arrive in the next time slot. As each vehicle travels with stable speed in the coverage of the RSU, the total distance traveled during each time slot is identical. If we consider the total distance traveled during one time slot as a small region, then the whole coverage of the RSU can be regarded as virtually divided into small pieces. The number of small regions is equal to the number of total time slots traveling within the RSU's coverage.

According to the wireless model, since the available transmission rate is mainly dominated by the relative distance $d$ 
to the RSU, the transmission rate for each region can be represented by a vector $\mathcal{W}=\left\{w_{1}, w_{2}, \ldots, w_{N_{T}}\right\}$, where $N_{T}$ is total number of small regions and $N_{T}=T_{s} / \Delta t$. The value of each element $w_{i}$ can be easily obtained by the wireless model based on the relative distance to RSU.

Since the wireless channel is broadcast and shared in nature, in each time slot, it only allows at most one vehicle to transmit. Let $t=1$ represents the first time slot, for the $t$-th time slot, the transmission scheduling decision for each small region can be represented by a vector:

$$
\mathcal{A}(t)=\left\{a_{1}(t), a_{2}(t), \ldots, a_{N_{T}}(t)\right\},
$$

where

$$
a_{i}(t)= \begin{cases}1, & \text { vehicle in the } i \text {-th region is assigned, } \\ 0, & \text { vehicle in the } i \text {-th region is NOT assigned. }\end{cases}
$$

There is a constraint $\sum_{i=1}^{N_{T}} a_{i}(t) \leq 1$ for the vector $\mathcal{A}(t)$, which means that at most one vehicle will be assigned to transmit for slot $t$.

With multiple vehicles competing for the limited wireless resources, the problem is formulated as finding the best time slot allocation for a total time period $T$ to schedule the transmission, such that the total utility is maximized. Therefore, we formulate the utility maximization problem (UMP) as:

$$
\begin{aligned}
\text { UMP: } \max & \sum_{i=1}^{N_{c}} U_{i}+\sum_{n=1}^{T} \mathbf{E}\left(U_{n}\right), \\
\text { s.t. } & a_{j}(t) \in\{0,1\}, j=1,2, \ldots, N_{T}, \\
& \sum_{l=1}^{N_{T}} a_{l}(t) \leq 1,
\end{aligned}
$$

where, $\sum_{i=1}^{N_{c}} U_{i}$ is the total utility of the $N_{c}$ vehicles currently in the RSU's transmission range, and $\sum_{n=1}^{T} \mathbf{E}\left(U_{n}\right)$ is the expectation of total utility achieved of future arrival vehicles in the next $T$ time slots.

For the current $N_{c}$ vehicles in the RSU's transmission range, the $i$-th vehicle is supposed to arrive at $t_{i}$, and the decision vector $\left[a_{1}\left(t_{i}\right), a_{2}\left(t_{i}+1\right), \ldots, a_{N_{T}}\left(t_{i}+N_{T}-1\right)\right]$ indicates whether the vehicle is allocated to transmit. Then the achieved utility $U_{i}$ of the $i$-th vehicle is obtained by the utility function $F_{u}$ based on the total throughput:

$$
U_{i}=F_{u}\left(\sum_{j=1}^{N_{T}} a_{j}\left(t_{i}+j-1\right) w_{j} \Delta t\right)
$$

Since the future arrival information is unavailable, only the expectation of utility achieved by future arrival vehicle can be calculated. For the future arrived vehicle, the encoding technique of the video to transmit can be selected from the set of $N_{e}$ encoding techniques with certain probability $P_{e}$. Assume that there is a new vehicle arrives in the next time slot with probability $P_{a}$, then the expected utility $\mathbf{E}\left(U_{n}\right)$ for the new arrival vehicle can be calculated by:

$$
\mathbf{E}\left(U_{n}\right)=P_{e} P_{a} \sum_{i=1}^{N_{e}} F_{u}\left(\sum_{j=1}^{N_{T}} a_{j}\left(t_{n}+j-1\right) w_{j} \Delta t\right) .
$$

Apparently, the formulated UMP problem is an integer programming problem [28]. Since the variable $a(t)$ in UMP can be only 0 or 1 , the formulated UMP is a $0-1$ programming problem and it is NP-hard according to [29]. For each time slot, $N_{v}$ vehicles in the RSU's transmission range correspond to $N_{v}$ choices for the scheduler. As the variable $a(t)$ must be solved sequentially, the decision at the current time slot $a(t)$ will affect the future decision. With the scheduling for $T$ time slots, the computation complexity is $O\left(\left(N_{v}\right)^{T}\right)$.

Meanwhile, without the future arrival information, it is even more difficult to obtain the optimal solution. Therefore, in the next step we first consider how to obtain the optimal solution with the assumption that the future arrival information is known already, which is not practical and serves as a benchmark. Then we consider how to devise an heuristic algorithm which is only based on the current vehicle information to achieve high utility.

\section{Algorithm Design}

\section{A. Optimal Solution}

With the assumption that the RSU has the knowledge of future vehicle arrival pattern, the formulated problem can be translated into a finite state sequential decision problem.

In our problem, the goal is to obtain the maximum utility achieved in $T$ time slots. For each time slot $t$, we define state $S_{t}$ to represent the current status of $N_{v}$ vehicles in the RSU's coverage:

$$
S_{t}=\left(V_{1}, V_{2}, \ldots, V_{N_{v}}\right),
$$

where $V_{i}$ is the information set of the $i$-th vehicle in the coverage and is defined as:

$$
V_{i}=\left\{I_{i}, P_{i}, u_{i}^{T}, D_{i}^{S}, D_{i}^{R}\right\},
$$

which includes the vehicle ID $I_{i}$, the current position $P_{i}$, the utility type $u_{i}^{T}$, the total amount of data $D_{i}^{S}$ to transmit, and the amount of remaining data $D_{i}^{R}$.

For each time slot, we only need to determine which vehicle is allocated to transmit. Thus the actions is defined as:

$$
A_{t}=a, a \in\left[1,2, \ldots, N_{v}\right] .
$$

If the action $A_{t}=i$, then the $i$-th vehicle is allocated to transmit the video data during the current slot.

For the $i$-th vehicle, we can calculate the current relative distance $d_{i}$ to the RSU according to its current position $P_{i}$. The transmission data rate $w_{i}$ can be estimated accordingly. Then the total amount of data that can be transmitted in the current time slot is obtained by:

$$
\Delta D=w_{i} \Delta t
$$

With the above amount of data transmitted in the current time slot, the amount of remaining data in the next time slot $D_{i}^{R+}$ is updated by:

$$
D_{i}^{R+}=D_{i}^{R}-\Delta D
$$

For the other vehicles, without the chance to transmit, their remaining amounts of the video do not change.

Since all the vehicles will move with distance $v \Delta t$, the information for each vehicle in the next time slot $V_{i}^{+}$needs to 
be updated accordingly. Only the vehicle that is allocated to transmit in the current time slot has to update the remaining amount of video. For the next time slot, the next state $S_{t}^{+}$can be represented by:

$$
S_{t}^{+}=\left(V_{1}^{+}, V_{2}^{+}, \ldots, V_{N_{v}}^{+}\right) .
$$

To evaluate how good the decision is, we define the reward $R_{t}$ for each action at each time slot $t$. Since the goal of our schedule algorithm is to achieve the maximum utility, the utility achieved by the current $N_{v}$ vehicles in the current time slot will be used as the reward. Therefore, the reward can be obtained by:

$$
R_{t}=\sum_{i=1}^{N_{v}}\left[F_{u}\left(D_{i}^{S}-D_{i}^{R+}\right)-F_{u}\left(D_{i}^{S}-D_{i}^{R}\right)\right] .
$$

The total reward achieved by all the time slots is the summation of the reward of each time slot. Then the problem can be formulated as the maximization of the long term reward during the whole running time:

$$
\begin{aligned}
\max & \sum_{t=1}^{T} R_{t}, \\
\text { s.t. } & A_{t} \in\left[1, N_{v}\right], t \in[1, T] .
\end{aligned}
$$

Note that, for each time slot, if we always take the action which can bring the highest reward for the current slot, such a greedy approach cannot guarantee the long-term highest utility. For instance, as shown in Fig. 3, within one time slot, the vehicle $V_{s}$ with SVC video will achieve 0 utility as the amount of transmitted data cannot even decode the base layer, while another vehicle $V_{c}$ with CS video can always achieve certain amount of utility. With higher utility to achieve, $V_{c}$ will be allocated to transmit. However, if $V_{s}$ can transmit for three consecutive time slots, the reward it can bring will be higher than that of $V_{c}$. So, the greedy algorithm which results in immediate optimum cannot guarantee the long-term optimum.

The global optimal solution of the above optimization problem can be solved by searching all the states. Obviously, the computational complexity is still $O\left(N_{v}^{T}\right)$, which is prohibitively high. Meanwhile, since the prior knowledge of future arrival is required, such a solution cannot be obtained in practice and it can only be considered as a benchmark offline algorithm and the performance upper bound.

\section{B. Max Utility Potential Algorithm}

Considering the high mobility and dynamic arrival of the vehicles, it is impractical to have future vehicle arrival information. Thus, an online heuristic algorithm should be devised without the prior knowledge of future arrival. In this section, based on the current vehicles' information, we design a simple yet effective algorithm to achieve high utility.

As discussed before, the immediate optimum of single slot cannot guarantee the long-term optimal results, and more consecutive time slots should be considered to improve the results. Therefore, for each vehicle, all the time slots before it leaves the RSU's transmission range can be considered to check the maximum potential of the achieved utility. Meanwhile, since

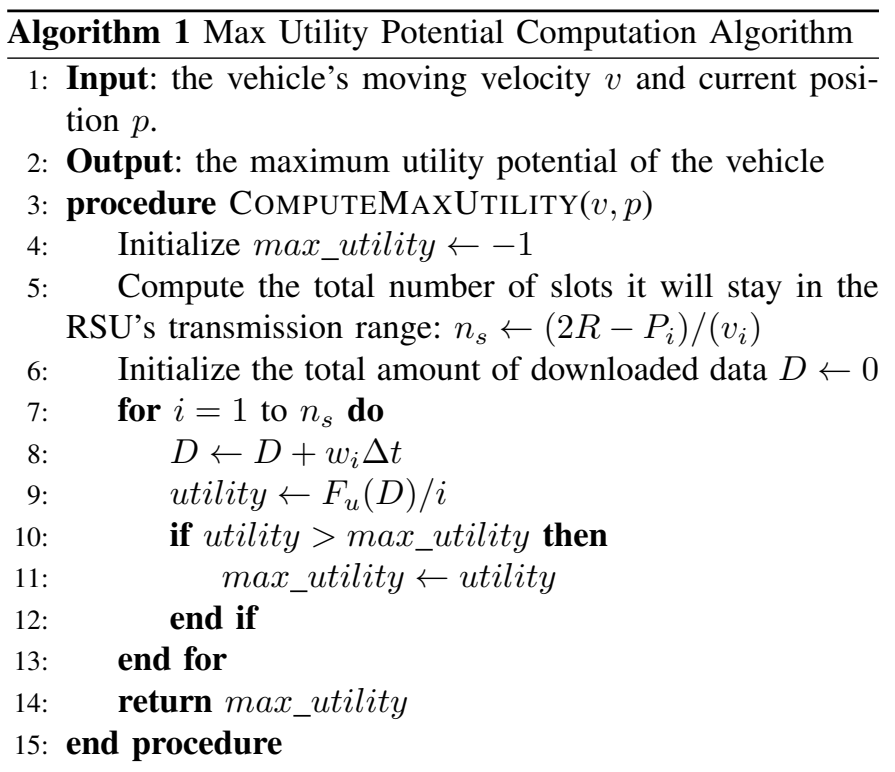

the future vehicle arrival is unavailable and quite difficult to predict, only the vehicles currently in the RSU's coverage will be taken into consideration.

For each vehicle, with the position $P_{i}$ and the moving velocity $v_{i}$, the RSU can accurately predict how long the vehicle will stay in the transmission range. Then the total number of time slots that the vehicle will stay in the RSU's transmission range can be calculated:

$$
n_{s}=\frac{2 R-P_{i}}{v_{i} \Delta t} .
$$

The maximum possible utility achieved for each vehicle can be estimated if the vehicle can always be allocated as long as it is inside the RSU's transmission range. Then the average utility potential in the next $n$ slots $U_{n}$ is defined as the total amount of utility obtained during the next $n$ slots over $n$, which is computed as:

$$
U_{n}=\frac{F_{u}\left(\sum_{i=1}^{n} w_{i} \Delta t\right)}{n} .
$$

If there is only a small amount of data left to be transmitted, only a fewer number of slots are needed. So if too many future time slots are allocated to the vehicle, many will be wasted. In this case, as the total amount of utility achieved in the future will not change, with more time slots, the average utility potential will be decreased. Besides, for certain types of utility function, with more data downloaded, the increasing rate of achieved utility will slow down. Therefore, the maximum utility potential $\max \left(U_{1}, U_{2}, \ldots, U_{n_{s}}\right)$ should be computed for the scheduling, which is shown in lines 6 to 12 of Algorithm 1.

The computation of utility potential can be considered as to find the vehicle which can bring the highest utility-tothroughput ratio in the following couple of slots till it leaves the RSU coverage. For a discrete utility type, the computation is to find the steepest stair, while for a continuous utility function, it is close to find the maximum slope.

With the utility potential computed, all the vehicles in the RSU's transmission range will be sorted according to the 
utility potential. The vehicle with the highest utility potential will be selected to transmit during the current time slot.

\section{Discussion}

From Algorithm 1, it can be noticed that the calculation of utility potential for each vehicle will consider at most $N_{T}$ time slots. According to the traffic model, given the vehicle density $k$, the maximum number of vehicles $N_{v}$ currently in the RSU's transmission range can be estimated by (3). Therefore, for each time slot, there will be at most $N_{v}$ vehicles to be considered for the utility potential. Then the worst-case computation complexity can be derived as $O\left(N_{v} N_{T}\right)$, and thus the proposed algorithm is simple enough to run in real time.

The estimation of the utility potential can be considered as the slope between the amount of transmitted data and the utility, which is shown in Fig. 4(a). Although the proposed algorithm works in a myopic way, it still can achieve good performance without taking the future prediction into consideration. Supposing that one vehicle has been selected to transmit, the future vehicle arrival can be categorized in two scenarios:

- In the next several future time slots, the utility potential of incoming vehicles cannot exceed the current selected vehicle. In this case, the proposed algorithm definitely makes the best decision.

- The future arrival vehicle will achieve a higher utility potential. If shortly after the current slot, there is one incoming vehicle with a higher utility potential, then our algorithm will select the new vehicle to transmit. Within a short time period, the data transmitted by the previous vehicle is very limited, and thus any possible throughput wastage is limited. For a vehicle with stair-case type of utility functions (e.g., for SVC or H.264 video), once it is served, the utility potential for the selected vehicle will become higher, which is shown in Fig. 4(b). With more amount of video data transmitted, the slope also becomes larger for flows with continuous utility functions. Therefore, there is a very small probability that the utility potential of the future arrival vehicles can exceed the current selected vehicle, so we can largely avoid the situation of wasting throughput.

The performance of the proposed utility potential based approach is affected by the utility function. If the vehicle with a stair-case utility function is assigned to transmit for a few time slots, the transmitted video data may not be able to be decoded, therefore 0 utility is achieved, and the throughput is wasted. While for the continuous type utility function, even if the vehicle is only allocated to transmit for one slot, the limited throughput can still achieve certain utility. Therefore, if there are more vehicles with continuous utility functions, the total achieved utility will be closer to the optimal result.

\section{Performance Evaluation}

\section{A. Simulation Setup}

To evaluate the performance of the proposed scheduling algorithm, we implemented simulation with NS-3 and conducted simulations with real video traces. Two video traces

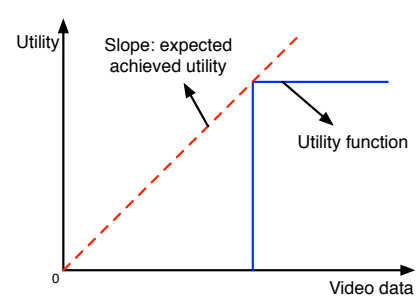

(a) Utility Potential at time $t_{0}$

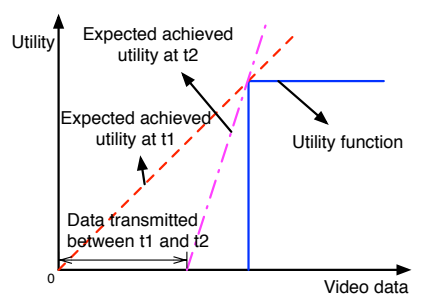

(b) Utility Potential at time $t_{1}$
Fig. 4. Illustration of potential achieved utility.

TABLE I

VIDEO ENCODING CONFIGURATIONS

\begin{tabular}{|c|c|c|c|}
\hline Video & Codec & Avg. Bitrate & Avg. PSNR \\
\hline \hline \multirow{5}{*}{ Foreman } & H.264 & $726.8 \mathrm{kbps}$ & $39.5 \mathrm{~dB}$ \\
\cline { 2 - 4 } & SVC layer 1 & $98.6 \mathrm{kbps}$ & $29.2 \mathrm{~dB}$ \\
& SVC layer 2 & $158 \mathrm{kbps}$ & $32.89 \mathrm{~dB}$ \\
& SVC layer 3 & $374 \mathrm{kbps}$ & $36.8 \mathrm{~dB}$ \\
\cline { 2 - 4 } & CS & Avg. PSNR $\approx 2 \times$ Bitrate $^{0.38}+8.51$ \\
\hline \hline \multirow{3}{*}{ Football } & H.264 & $603.31 \mathrm{kbps}$ & $33.75 \mathrm{~dB}$ \\
\cline { 2 - 4 } & SVC layer 1 & $537.9 \mathrm{kbps}$ & $29.16 \mathrm{~dB}$ \\
& SVC layer 2 & $958.2 \mathrm{kbps}$ & $33.27 \mathrm{~dB}$ \\
& SVC layer 3 & $1,408.7 \mathrm{kbps}$ & $35.53 \mathrm{~dB}$ \\
\cline { 2 - 4 } & CS & \multicolumn{2}{|c|}{ Avg. PSNR $\approx 0.51 \times$ Bitrate $^{0.53}+14.62$} \\
\hline
\end{tabular}

Foreman and Football [30], which have 300 and 260 frames respectively, are used in our work. The videos are encoded with H.264, SVC and CS techniques, and the encoding configurations are listed in Table I. Other types of encoding techniques and utility functions can also be supported by our algorithm.

The settings of simulation parameters are shown in Table II. Based on the traffic flow model, the free-way speed is always impossible to achieve. For different simulation scenarios, the actual vehicle speed is set between $60 \mathrm{~km} / \mathrm{h}$ to $130 \mathrm{~km} / \mathrm{h}$. With the assumption that the wireless data rate is mainly determined by the relative distance, the wireless network achievable data rate is estimated according to [31], [32]. The wireless configuration is shown in Table III.

To better understand the performance of the proposed algorithm, we compare the results with the D*S/R algorithm proposed in [1], and two other simple schemes, the greedy algorithm which always allocates the wireless resources to the vehicle with the highest data rate, and the first come first service (FCFS) scheme which serves the vehicle that will leave the RSU's coverage first. We run the simulation with several different scenarios. For each scenario, we conduct 100 runs with different random seeds to obtain the average, and each run will last for 10 to 10,000 time slots.

\section{B. Evaluation Metrics}

In order to maximize the total achieved utility, the following metrics are used for evaluation purpose,

- Average Utility: defined as the average utility achieved by all the vehicles during the total time slots.

- Wasted Throughput Ratio: defined as the amount of throughput which does not contribute to any utility over the total throughput. 

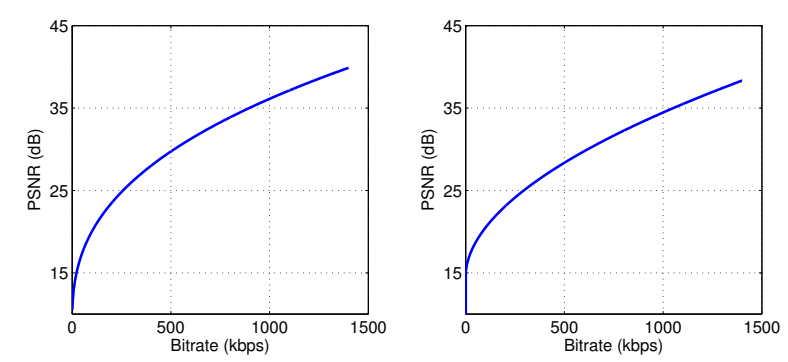

Fig. 5. Bitrate and PSNR of Compressed sensing video.

TABLE II

Simulation SETUP

\begin{tabular}{|c|c|}
\hline Parameter & Value \\
\hline RSU transmission range & $300 \mathrm{~m}$ \\
Traffic jam density $k_{\text {jam }}$ & $120 \mathrm{veh} / \mathrm{km} / \mathrm{lane}$ \\
Number of lanes & 2 \\
Free-way speed $v_{f}$ & $160 \mathrm{~km} / \mathrm{h}$ \\
\hline
\end{tabular}

- Jain's Fairness Index: calculated based on the achieved utilities from all the vehicles to evaluate the scheduling fairness.

\section{Simulation Results}

First, we evaluate the performance gap between solutions from the proposed algorithm to the optimal solution. As mentioned before, the computation complexity is very high for the optimal solution. To reduce the computation load, we set the total time slots $T=10$, and the duration of each time slot is $\Delta t=1$ second with a very low vehicle density (5 vehicles per kilometer per lane). With the above setting, each run of optimal solution can be solved in around 10 minutes.

The results are shown in Fig. 6. The average utility achieved versus the vehicle speed is plotted with $95 \%$ confidence interval in Fig. 6(a). Obviously, the optimal solution achieves the best performance. As the optimal solution always searches all possible allocations to find the best solution, the performance affected by the randomness is limited. Although the proposed algorithm achieves less average utility than the optimal solution, small variances can be achieved as well. The greedy algorithm always chooses the vehicle with the highest data rate to transmit, which brings high utility. In the implementation, the deadline of $\mathrm{D} * \mathrm{~S} / \mathrm{R}$ algorithm for each vehicle is considered as the time it leaves the transmission range. Thus, the vehicle leaving the transmission range sooner will be given a higher priority to transmit. Meanwhile, the low data rate caused by relatively long distance to the RSU leads to low average utility. Similar to the D*S/R algorithm, FCFS always selects the vehicle closest to the transmission edge to transmit, and the worst performance is achieved, compared to the other solutions.

For all the algorithms, the average achieved utility decreases with the increment of the vehicle speed. This is because when the vehicle speed is faster, the sojourn time is shorter with the fixed transmission range of RSU. Therefore, the total throughput for each vehicle will be be reduced, which leads to a smaller average utility.
TABLE III

WIRELESS NETWORK SETUP

\begin{tabular}{|c|c|}
\hline Distance range $(\mathrm{m})$ & Data Rate $(\mathrm{Mbps})$ \\
\hline$[0,85]$ & 3 \\
{$[85,135]$} & 6 \\
{$[135,215]$} & 12 \\
{$[215,300]$} & 24 \\
\hline
\end{tabular}

The result of the percentage of the wasted throughput is plotted in Fig. 6(b) with 95\% confidence interval as well. From the results, we can find that the proposed algorithm can reach similar low bandwidth wastage (around 3\%) and small variances to the optimal solution. As the proposed algorithm always tries to find the vehicle with the highest potential to transmit, the throughput will not be wasted much. In this scenario, since we only run the simulation for 10 slots, the data requested during the last several slots may be wasted, as a significant percentage of vehicles are still within the coverage of RSU when the simulation was ended. For the other three algorithms, the wasted throughput is much higher, and the percentage of wastage increases linearly with the increment of vehicle speed. With a higher speed, since the sojourn time becomes less, the probability that a vehicle cannot finish the transmission becomes higher which leads to a higher percentage of wasted throughput.

Next, we conduct the simulations with more time slots. In this case, the time slot duration is set to $\Delta t=0.1 \mathrm{~s}$ to ensure that there is at most one vehicle arrival during each time slot. The total time slots is set to $T=10,000$. The optimal solution cannot be obtained for comparison as the total amount of time slots is too large, such that the computation load has already exceeded our computation capacity. In order to evaluate the performance under different vehicle densities, which are set to 10 and 30 vehicles per kilometer per lane respectively. For each scenario, all vehicles run with same average speed with different variations. The results are plotted in Figs. 7 and 8 with $95 \%$ confidence interval.

Figures 7(a) and 8(a) show the results of the average achieved utility. From the figures, it can be noticed that performance variances for all the algorithms have been greatly reduced. According to our simulation, when the simulation runs for a long time, the performance of each algorithm will finally converge. Compared to the previous case, the results show the similar trend. Less average utility can be obtained due to the higher traffic. With more vehicles, there will be more competitions for the limited wireless resources. As a result, the average throughput for each vehicle will be reduced and will lead to less average utility. By considering the possible highest utility potential, our utility maximization based algorithm can outperform the other algorithms.

In Figs. 7(b) and 8(b), the results of the percentage of wasted throughput are shown. With the long running time, the proposed algorithm wastes very limited amount of throughput. Theoretically, the proposed algorithm only let the vehicles which can gain utility to transmit, so none of the throughput should be wasted. However, with the stair-type utility function, the throughput may be wasted. For instance, as shown in Fig. 3, during $t_{3}$, the residual base layer data and part of 


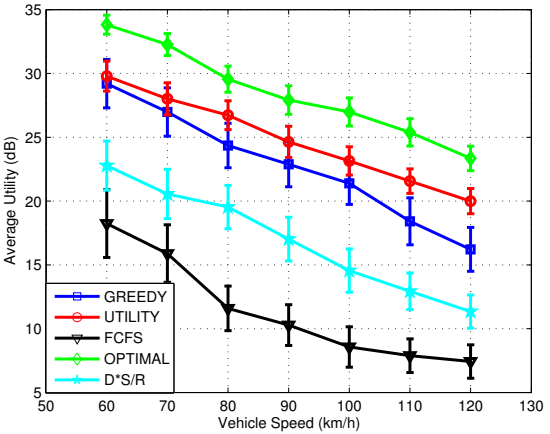

(a) Average achieved utility.

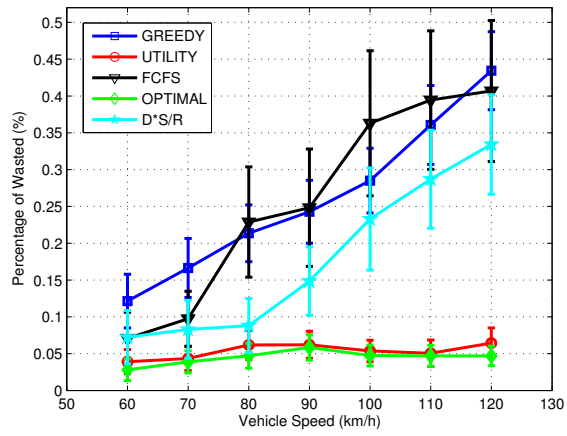

(b) Percentage of wasted throughput.

Fig. 6. Results of Case 1, with vehicle density $k=5$ vehicles per kilometer per lane, and $T=10$ time slots.

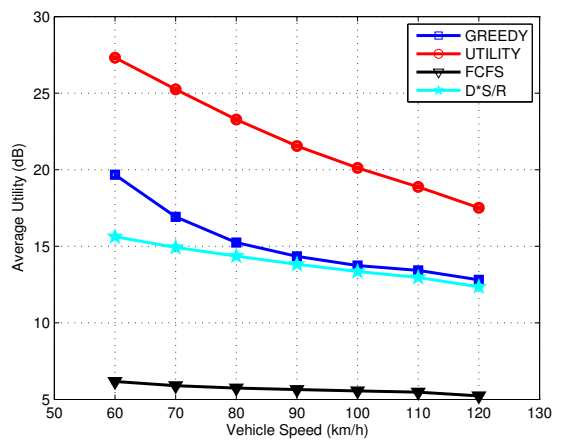

(a) Average achieved utility.

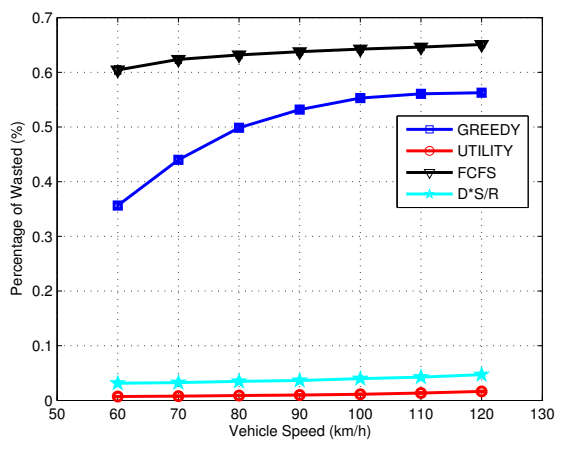

(b) Percentage of wasted throughput.

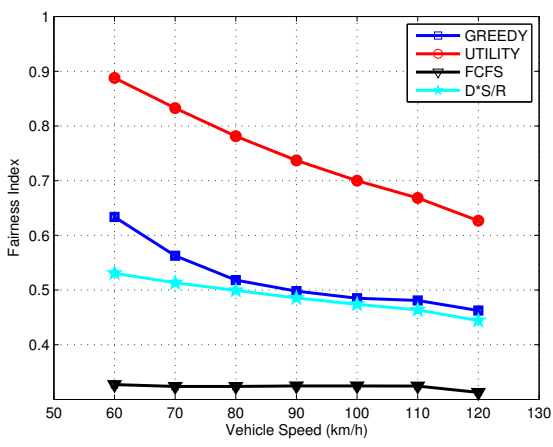

(c) Fairness index.

Fig. 7. Results of Case 2, with vehicle density $k=10$ vehicles per kilometer per lane, and $T=10,000$ time slots.

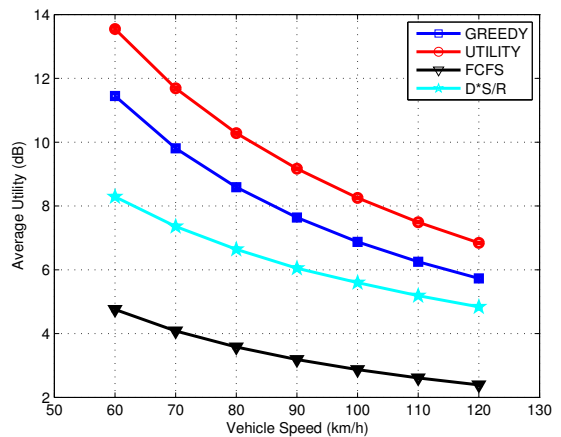

(a) Average achieved utility.

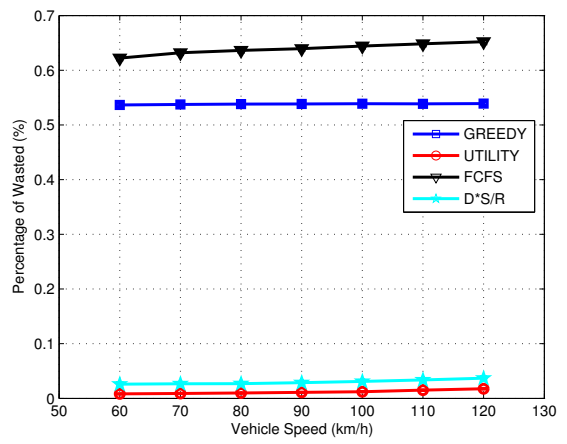

(b) Percentage of wasted throughput.

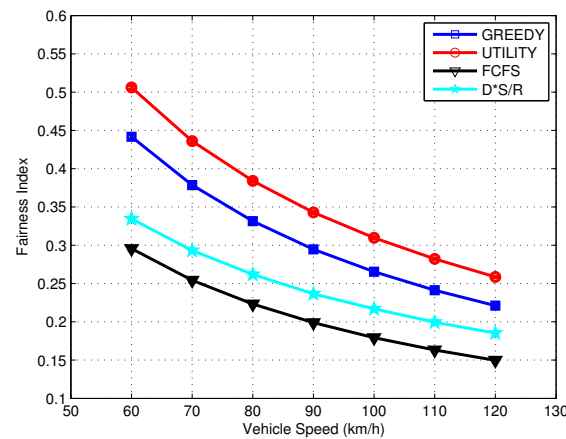

(c) Fairness index.

Fig. 8. Results of Case 3, with vehicle density $k=30$ vehicles per kilometer per lane, and $T=10,000$ time slots

enhancement layer data will be transmitted simultaneously. Since the transmitted enhancement layer is not enough to decode, the bandwidth to transmit the enhancement layer data is wasted. Meanwhile, the performance does not change much with different vehicle speed. This can be explained that the estimation of the potential achieved utility is independent of the vehicle speed, thus it does not affect the performance.

The results of the fairness index are shown in Figs. 7(c) and $8(\mathrm{c})$. It can be noticed that the trend of the fairness index is similar to the average throughput. With the increment of vehicle speed, the average utility achieved will decrease. The lower average utility implies that more vehicles cannot achieve any utility, which leads to a lower fairness index. Therefore, as the proposed algorithm can achieve a higher average utility, the schedule fairness can be improved at the same time.

Finally, we conducted the simulations with the change of vehicle density. We still set the time slot duration to $0.1 \mathrm{~s}$ and run the simulation with 10,000 time slots. According to the traffic model, the vehicle speed can be estimated by (1). The results are plotted with 95\% confidence interval and shown in 


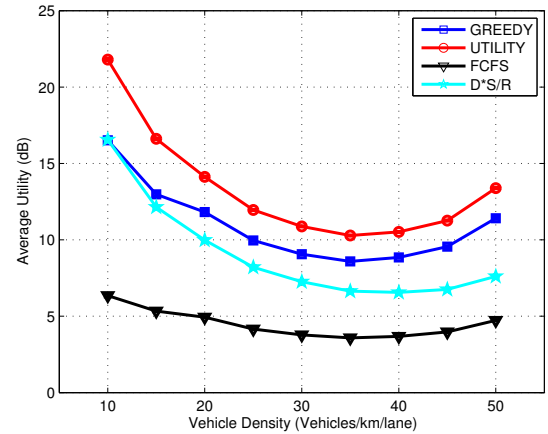

(a) Average achieved utility.

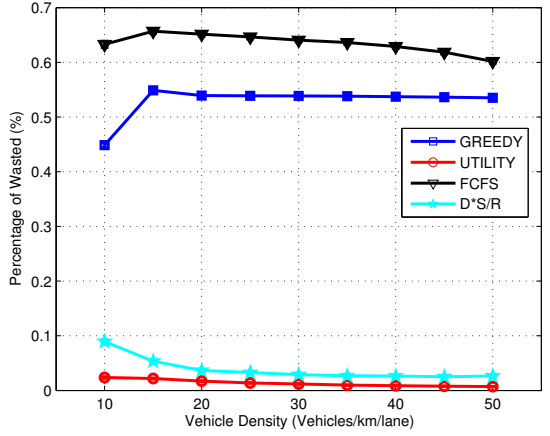

(b) Percentage of wasted throughput.

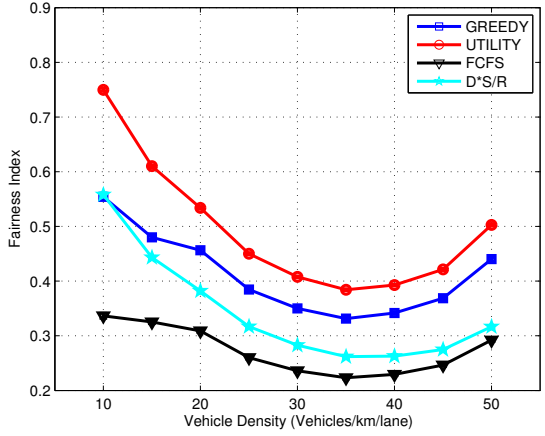

(c) Fairness index.

Fig. 9. Results of Case 4, with $T=10,000$ time slots.

Fig. 9.

Figure 9(a) shows the average achieved utility. Different from the previous results, when the vehicle density and the vehicle speed change simultaneously, the performance results do not change monotonically. With a low vehicle density, the low competition will bring relative high throughput. Thus a higher average utility will be achieved correspondingly. However, when the vehicle density reaches a certain level, according to (1), the vehicle speed will be decreased a lot. Therefore, each vehicle will have a longer sojourn time to improve the throughput.

From Fig. 9(b), we can still achieve low throughput wastage with the proposed algorithm. As mentioned before, the estimation of the utility potential is independent with the traffic arrival rate or density. Therefore, a low throughput wastage can always be guaranteed. $\mathrm{D} * \mathrm{~S} / \mathrm{R}$ takes both the deadline and throughput into consideration, which can reduce the throughput wastage as well. The greedy and FCFS did not consider whether the throughput can bring certain utility, thus more than half of the throughput was wasted.

At last, we show the fairness index in Figure 9(c). As explained before, the fairness index has a similar trend to the average achieved utility. With the higher average utility achieved, the proposed algorithm can outperform the other algorithms in terms of better fairness.

\section{CONCLUSIONS}

In this paper, the problem of multimedia transmission scheduling among multiple vehicles over the Drive-thru Internet has been investigated. The utility model was devised to map the throughput to the user's satisfaction level. First, the scheduling problem was formulated as an optimization problem to maximize the total achieved utility. Since the optimization problem is NP-hard, the problem was then converted to a finite state decision problem and solved by a searching algorithm which is served as the performance upper bound. A heuristic algorithm based on the utility potential was proposed to obtain the solution in real time with high performance. Finally, we implemented and conducted extensive simulations to evaluate the performance.

There are still open issues to investigate in the future. First, the wireless model should take the channel fading and shadowing into consideration to make it more practical. Second, the vehicles may move with varying speed. Last but not least, we only consider one RSU scenario, how to schedule the transmission when vehicles drive through multiple RSUs need to be investigated.

\section{REFERENCES}

[1] Y. Zhang, J. Zhao, and G. Cao, "Service scheduling of vehicle-roadside data access," Mobile Networks and Applications, vol. 15, no. 1, pp. 8396, 2010.

[2] M. H. Cheung, F. Hou, V. W. Wong, and J. Huang, "DORA: Dynamic optimal random access for vehicle-to-roadside communications," IEEE J. Sel. Areas Commun., vol. 30, no. 4, pp. 792-803, 2012.

[3] T. H. Luan, X. Ling, and X. Shen, "MAC in motion: impact of mobility on the MAC of drive-thru internet," IEEE Trans. Mobile Comput., vol. 11 , no. 2 , pp. 305-319, 2012.

[4] F. Soldo, C. Casetti, C. Chiasserini, and P. A. Chaparro, "Video streaming distribution in VANETs," IEEE Trans. Parallel Distrib. Syst., vol. 22, no. 7, pp. 1085-1091, 2011.

[5] M. Asefi, J. W. Mark, and X. Shen, "A mobility-aware and qualitydriven retransmission limit adaptation scheme for video streaming over VANETs," IEEE Trans. Wireless Commun., vol. 11, no. 5, pp. 1817$1827,2012$.

[6] A. Torres, C. T. Calafate, J.-C. Cano, P. Manzoni, and Y. Ji, "Evaluation of flooding schemes for real-time video transmission in VANETs," Ad Hoc Networks, 2014.

[7] F. Naeimipoor and A. Boukerche, "A hybrid video dissemination protocol for VANETs," in IEEE ICC'14, 2014, pp. 112-117.

[8] J.-S. Park, U. Lee, S. Y. Oh, M. Gerla, and D. S. Lun, "Emergency related video streaming in VANET using network coding," in $A C M$ VANET'06, 2006, pp. 102-103.

[9] A. Vinel, E. Belyaev, K. Egiazarian, and Y. Koucheryavy, "An overtaking assistance system based on joint beaconing and real-time video transmission," IEEE Trans. Veh. Technol., vol. 61, no. 5, pp. 2319-2329, 2012.

[10] K.-H. Liu, L. Cai, and X. Shen, "Multiclass utility-based scheduling for UWB networks," IEEE Trans. Veh. Technol., vol. 57, no. 2, pp. 1176$1187,2008$.

[11] "Network simulator 3," http://www.nsnam.org.

[12] W. L. Tan, W. C. Lau, O. Yue, and T. H. Hui, "Analytical models and performance evaluation of drive-thru internet systems," IEEE J. Sel. Areas Commun., vol. 29, no. 1, pp. 207-222, 2011.

[13] Y. Zhuang, J. Pan, V. Viswanathan, and L. Cai, "On the uplink MAC performance of a drive-thru internet," IEEE Trans. Veh. Technol., vol. 61, no. 4, pp. 1925-1935, 2012.

[14] J. J. Alcaraz, J. Vales-Alonso, and J. García-Haro, "Link-layer scheduling in vehicle to infrastructure networks: An optimal control approach," IEEE J. Sel. Areas Commun., vol. 29, no. 1, pp. 103-112, 2011.

[15] M. H. Cheung, F. Hou, V. W. Wong, and J. Huang, "Dynamic optimal random access for vehicle-to-roadside communications," in IEEE ICC'11, 2011, pp. 1-6. 
[16] N. Cheng, N. Lu, N. Zhang, X. Shen, and J. W. Mark, "Vehicular WiFi offloading: Challenges and solutions," Vehicular Communications (Elsevier), vol. 1, no. 1, pp. 13-21, 2014.

[17] A. E. Abdulla, Z. M. Fadlullah, H. Nishiyama, N. Kato, F. Ono, and R. Miura, "An optimal data collection technique for improved utility in UAS-aided networks," in IEEE INFOCOM'14, 2014, pp. 736-744.

[18] T. Ngo, H. Nishiyama, N. Kato, T. Sakano, and A. Takahara, "A spectrum- and energy-efficient scheme for improving the utilization of mdru-based disaster resilient networks," IEEE Transactions on Vehicular Technology, vol. 63, no. 5, pp. 2027-2037, June 2014.

[19] J.-B. Hwang et al., "Effective video multicast using SVC with heterogeneous user demands over TDMA-based wireless mesh networks," IEEE Trans. Mobile Comput., vol. 12, no. 5, pp. 984-994, 2013.

[20] J. Chen, S. He, Y. Sun, P. Thulasiraman, and X. Shen, "Optimal flow control for utility-lifetime tradeoff in wireless sensor networks," Computer Networks, vol. 53, no. 18, pp. 3031 - 3041, 2009.

[21] J. Chen, W. Xu, S. He, Y. Sun, P. Thulasiraman, and X. Shen, "Utility-based asynchronous flow control algorithm for wireless sensor networks," Selected Areas in Communications, IEEE Journal on, vol. 28, no. 7, pp. 1116-1126, September 2010.

[22] D. Bethanabhotla, G. Caire, and M. J. Neely, "Utility optimal scheduling and admission control for adaptive video streaming in small cell networks," in IEEE ISIT'13, 2013, pp. 1944-1948.

[23] L. Zhou, H. Wang, S. Lian, Y. Zhang, A. Vasilakos, and W. Jing, "Availability-aware multimedia scheduling in heterogeneous wireless networks," IEEE Trans. Veh. Technol., vol. 60, no. 3, pp. 1161-1170, 2011.

[24] J. D. Fricker and R. K. Whitford, Fundamentals of Transportation Engineering: A Multimodal Systems Approach. Pearson Prentice Hall, 2004.

[25] K. Lin, W.-L. Shen, C.-C. Hsu, and C.-F. Chou, "Quality-differentiated video multicast in multirate wireless networks," IEEE Trans. Mobile Comput., vol. 12, no. 1, pp. 21-34, 2013.

[26] S. Xiang and L. Cai, "Transmission control for compressive sensing video over wireless channel," IEEE Trans. Wireless Commun., vol. 12, no. 3, pp. 1429-1437, 2013.

[27] J. He, L. Duan, F. Hou, P. Cheng, and J. Chen, "Multiperiod scheduling for wireless sensor networks: A distributed consensus approach," IEEE Trans. Signal Process., vol. 63, no. 7, pp. 1651-1663, April 2015.

[28] "Integer programming," http://en.wikipedia.org/wiki/Integer_ programming.

[29] “Karp's 21 NP-complete problems," http://en.wikipedia.org/wiki/Karp\% 27s 21_NP-complete_problems.

[30] "Video Test Media," https://media.xiph.org/video/derf.

[31] J. Henry, CCNP Wireless CUWSS Quick Reference. Pearson Education, 2010.

[32] C. Campolo and A. Molinaro, "Data rate selection in WBSS-based IEEE 802.11 p/WAVE vehicular ad hoc networks," in IEEE CSNDSP'10, 2010, pp. $412-416$.

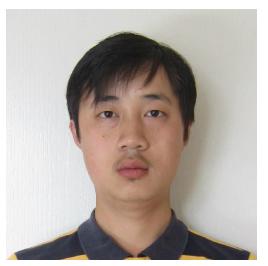

Min Xing is currently a Ph.D candidate in the Department of Electrical and Computer Engineering, University of Victoria, British Columbia, Canada. He eceived B.S. degree in Computer Science from Soochow University, Suzhou, Jiangsu in 2007, and M.S. degree in Software Engineering from Tongji University, Shanghai, China in 2010. His current research interest is multimedia transmission over heterogeneous wireless networks.

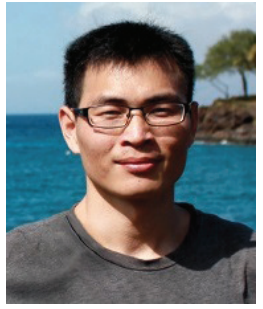

Jianping $\mathrm{He}$ is currently a postdoctoral research fellow in both the State Key Laboratory of Industrial Control Technology at Zhejiang University and the Department of Electrical and Computer Engineering at University of Victoria. He received the $\mathrm{Ph} . \mathrm{D}$. degree of Control Science and Engineering at Zhejiang University, Hangzhou, China. He is a member of Networked Sensing and Control group $(\mathrm{Nesc})$. His research interests include the control and optimization in sensor networks and cyberphysical systems, the scheduling and optimization in vehicular networks and social networks, and the investment decision in financial market and electricity market.

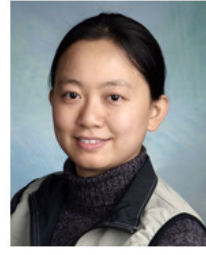

Lin Cai (S'00-M'06-SM'10) received her M.A.Sc. and $\mathrm{PhD}$ degrees (awarded Outstanding Achievement in Graduate Studies) in electrical and computer engineering from the University of Waterloo, Waterloo, Canada, in 2002 and 2005, respectively. Since 2005, she has been an Assistant Professor and then an Associate Professor with the Department of Electrical \& Computer Engineering at the University of Victoria. Her research interests span several areas in wireless communications and networking, with a focus on network protocol and architecture design supporting emerging multimedia traffic over wireless, mobile, ad hoc, and sensor networks.

She has been a recipient of the NSERC Discovery Accelerator Supplement Grant in 2010, and the best paper awards of IEEE ICC 2008 and IEEE WCNC 2011. She has served as a TPC symposium co-chair for IEEE Globecom' 10 and Globecom'13, and the Associate Editor for IEEE Transactions on Wireless Communications, IEEE Transactions on Vehicular Technology, EURASIP Journal on Wireless Communications and Networking, International Journal of Sensor Networks, and Journal of Communications and Networks (JCN). 